Once our new strategy was approved by the Board an extensive community engagement plan was put in place. This provided opportunities to promote our services at high profile events where we invited people with influence from many different backgrounds, asking for their ongoing support. Further community engagement included a wide ranging public awareness campaign.

Conclusion Overall the involvement of our community in preparation and dissemination of our new strategy has proved very worthwhile. To ensure ongoing inclusion of our community in our plans a new hospice advisory group PATH (People Advising the Hospice) has been established.

\section{P-263 HOSPICE OF THE FUTURE: PARTNERING WITH THE NEXT GENERATION}

Lonan Oldam, Lottie Morris, Giovanna Cruz, Gillian Street, Hollie Quaye, Lynsey Christian, Anne Mills. Hospice Isle of Man, Douglas, Isle of Man

\subsection{6/bmispcare-2019-HUKNC.285}

Background Co-design of services is increasingly recognised as an important means of ensuring development of people-centred services. Young people and adolescents are not often consulted when designing adult services but Hospice Isle of Man (IoM) aimed to change that.

Aims

- To engage with young people.

- To explore their attitudes towards death, dying, bereavement and Hospice.

Methods Hospice IoM's public engagement strategy started with 'Listening Events' in seven community settings which obtained views towards Hospice. Males and young people were under-represented so events were offered in secondary schools. A short session on end-of-life and palliative care was incorporated with activities to ascertain understanding of Hospice, uncover what was most valued and obtain recommendations for Hospice's future.

Students were asked 'What do you know about Hospice'? 'If you or a family member became seriously unwell and needed Hospice services, what would be most important to you?' and 'How could we make Hospice a better service?' Responses were made on post-it notes and analysed using NVivo. Themes and sub-themes within responses were identified.

Results 203 students (105 males, 98 female) aged 11 to 16, participated. For the first question, the themes were purpose (sub-themes helping and caring), services (sick or illness) and users. It was clear that many young people knew nothing about Hospice. For the second questions on what matters themes 'were how you feel', 'support services', 'staff qualities', 'environment', 'loved ones' and 'entertainment/ activities'. For the third question, community activities and age-appropriate care feature with suggestions that Hospice should feature more prominently within the local community and online.

Conclusion Young people provided a wealth of information from their own perspective and appreciated being involved at this level. These findings will inform future Hospice strategies, and the engagement with youth may empower new relationships between Hospice and the Island's next generation.

\section{P-264 NEEDS ASSESSMENT FOR PERSON-CENTRED PALLIATIVE AND END OF LIFE CARE IN THE ISLE OF MAN}

Sarah M McGhee, Giovanna I Cruz, Gillian Street, Lonan Oldam, Frances Newman, Anne Mills. Hospice Isle of Man, Douglas, Isle of Man

\subsection{6/bmjspcare-2019-HUKNC.286}

Background Isle of Man (IOM) is a crown dependency with 83,000 residents. To provide an evidence-base for the strategy on person-centred, palliative and end of life care, Hospice Isle of Man carried out a needs assessment. Typically this involves quantitative and qualitative data describing services and identifying gaps. Many data sources used for this purpose in the UK (e.g. Hospice UK's PopNAT) are not available in the IOM so we compensated by extensive engagement with those working in relevant services and organisations as well as the public.

Aim To carry out a needs assessment on palliative and end of life care.

Methods Population demographics and weekly death data were processed to enable predictions of need for palliative and end of life care. Interviews were carried out with over 60 representatives of services and organisations involved in care for those with life limiting conditions, in elderly care, end of life or bereavement support. The questions were: 'What works well?', 'What does not work well?', 'What are the gaps?' and 'What might be solutions?' in palliative and end of life care. Adult members of the public, schoolchildren and college students were asked what would matter to them if using Hospice services to ensure a focus on person-centred care.

Results We discovered that the hospice was probably reaching around half of those who could benefit but users were very positive about it. Passion for Island services, but frustration with aspects of the system, was identified among professionals and some disjointedness among third sector groups. The majority of the data are currently being analysed and will be presented at Hospice UK's 2019 conference.

Conclusion Expert views of those working in the services and charities, supplemented by person-centred perceptions of the public and available data are shedding necessary light on the Island's needs for palliative and end of life care.

(Support provided by Manx Lottery Trust)

\section{P-265 EMPOWERING HOSPICE STAFF THROUGH INTRAPRENEURSHIP}

Rami Okasha, Will McLean. Children's Hospices Across Scotland, Edinburgh, UK

\subsection{6/bmjspcare-2019-HUKNC.287}

Evidence about the impact of staff skills and motivation on securing positive outcomes for children is well understood. Scotland's new health and social care standards speak to the importance of continual reflection on practice, and a willingness to create an empowered culture amongst staff teams, establishing that people experiencing care should 'benefit from a culture of continuous improvement.'

Since 2017, CHAS has embraced the concept of intrapreneurship and has partnered with a social enterprise, The Lens, to deliver an improvement programme designed to unlock and unleash the creativity and skills of staff, volunteers and 
supported families and use these to further improve the care of children with life-shortening conditions.

Staff, volunteers and families are encouraged to develop ideas that link to CHAS' strategic objectives of reaching more families and children and turn them into action. Annually CHAS Intrapreneurs pitch for a share of $£ 35 \mathrm{k}$ and winning ideas are selected by a panel of peer staff, volunteers and families. The judging panel is completely empowered to decide what projects will attract funding. Support is provided to build confidence in pitching, planning service improvements, and delivering change programmes. Projects which successfully attracted funding include:

- a partnership with an accessible playground so supported children can play outside;

- a programme of death cafés to facilitate community discussions about death and dying across Scotland;

- memory boxes for children of all ages giving families a gift they cherish;

- a communications project to better support children who do not verbalise;

- a paediatric palliative care network for community pharmacies;

- a creative space at one of our hospices for family members.

The delivery of winning ideas is built into the overall CHAS strategic plan and they are now demonstrably making a difference to hospice and palliative care in CHAS, improving the care we provide for children with life-shortening conditions.

\section{P-266 LIVING OUR VALUES - ONE CONVERSATION AT A TIME}

Sarah Ireland. St Margaret's Hospice, Somerset, UK

\subsection{6/bmispcare-2019-HUKNC.288}

Background In order to respond to the ever changing, complex environment, our hospice needed to use our values to help ground us in times of change.

Aims The on-going work is about creating a values based culture which enables and empowers staff to have important conversations, provides continuity in times of change and enables the hospice to respond positively to change and difference. We have taken an organisational development approach allowing the work to evolve overtime.

Methods We have purposefully and obviously integrated our values into key aspects of our patient-centred delivery. Values have been supported through practical and tangible projects:

- Creating and supporting a staff led Mental Health First Aiders Network compassionately creating safe spaces to listen;

- Delivering a creative and stretching three-day 'developing yourself as a manager' programme enabling a cross-hospice cohort to learn more about themselves and their teams;

- Initiating brave 'reflecting on our own death' conversations;

- Facilitating a values conversation at the whole organisation induction;

- Using the change in NMC standards to start using coaching approaches in our meaningful conversations;

- Engaging the Board of Trustees in their own values conversation.
Results Over the past year, we have reviewed our progress against our benchmark culture mapping, learning and adapting, looking for opportunities to develop new projects, such as a ground up process for identifying core competencies for the whole hospice and new approach for the induction of all volunteers which is accessible and inclusive.

'I will see my role as part of a much bigger service and value others more than I would have done before', New starter

Conclusions What works for us so far?:

- Starting with ourselves and being disruptive;

- Being informed and driven by the engagement and initiative of staff;

- Demonstrating that collaborating with others across silos gets things done.

\section{P-267 'MAKING QUALITY IMPROVEMENT THE DRIVING FORCE OF HOSPICE CULTURE'}

Tara Schrikker, Colin Twomey. St Wilfrid's Hospice, Eastbourne, UK

\subsection{6/bmjspcare-2019-HUKNC.289}

Background The hospice submitted its first Quality Accounts in 2013, with little engagement across teams. Quality Improvement Priorities (QIPs) form a key focus of this process. QIPs are now embedded and one example of an evolving quality improvement culture.

Aims To demonstrate:

- Quality Accounts as a key process to describe clinical activity;

- How QIPs lead the clinical agenda;

- Emergence of a quality improvement methodology culture.

Method When Quality Accounts were first submitted there was limited understanding of the process and implementation of QIPs.

In subsequent years there has been engagement of staff and volunteers in the creation of QIPs. The responsibilities for QIPs has been strengthened with a work plan which is discussed and monitored within People with Personal Experience (PPE ) and Clinical Effectiveness meetings, which reports to the Clinical Governance Committee.

PPE initiatives are completed for each QIP.

The Quality Improvement Lead has introduced Quality Assurance Forums for staff and volunteers. These aim to ensure high quality, safe and effective care is provided and builds a quality improvement culture.

Results The knowledge, engagement and use of Quality Accounts has increased significantly with their production involving many staff across teams, including board members.

QIPs are connected with the business plan, clinical strategy and values. They are used to develop goals for staff as part of their annual appraisal cycle. PPEs have shown staff engagement with service users and stakeholders and gained feedback to inform service development and innovation. The Quality Improvement Lead is now well established. Clinical Leaders are engaged with quality improvement methodology and its impact on care delivery.

Conclusions Embedding QIPs into different tiers of the organisation creates a common goal for employees and volunteers to 